Algebraic 83 Geometric Topology

Volume 1 (2001) 143-152

Published: 2 March 2001

ATG

\title{
Brunnian links are determined by their complements
}

\author{
Brian MANGUM \\ THEODORE STANFORD
}

\begin{abstract}
If $L_{1}$ and $L_{2}$ are two Brunnian links with all pairwise linking numbers 0 , then we show that $L_{1}$ and $L_{2}$ are equivalent if and only if they have homeomorphic complements. In particular, this holds for all Brunnian links with at least three components. If $L_{1}$ is a Brunnian link with all pairwise linking numbers 0 , and the complement of $L_{2}$ is homeomorphic to the complement of $L_{1}$, then we show that $L_{2}$ may be obtained from $L_{1}$ by a sequence of twists around unknotted components. Finally, we show that for any positive integer $n$, an algorithm for detecting an $n$-component unlink leads immediately to an algorithm for detecting an unlink of any number of components. This algorithmic generalization is conceptually simple, but probably computationally impractical.
\end{abstract}

AMS Classification 57M25; 57M27

Keywords Brunnian, knot, link, link equivalence, link complement

All spaces and maps will be taken to be piecewise-linear or smooth. A link $L$ is a closed one-dimensional submanifold of $S^{3}$ with a finite number of connected components. For convenience, we will assume an arbitrary orientation on a link $L$, and an arbitrary ordering of its components. Two links $L_{1}$ and $L_{2}$ are equivalent if there exists a homeomorphism of pairs from $\left(S^{3}, L_{1}\right)$ to $\left(S^{3}, L_{2}\right)$. Such a homeomorphism is required to preserve the orientation on $S^{3}$, but not required to preserve the orientation or ordering on the $L_{i}$. If two links $L_{1}$ and $L_{2}$ are equivalent, then their complements $S^{3}-L_{1}$ and $S^{3}-L_{2}$ are homeomorphic. A basic question is when the converse of this statement holds. For knots (links with a single component), this problem was solved by Gordon and Luecke, who showed that two knots are equivalent if and only if they have homeomorphic complements ([GL89]). For links with more than one component, there are many examples where this result fails. A simple way to construct such examples is to take any link $L_{1}$ with an unknotted component $K$, cut along the disk bounded by $K$ (which usually intersects some of the other components of $L_{1}$ ), twist one or more times, and reglue. The resulting 
link $L_{2}$ will have a complement homeomorphic to that of $L_{1}$, and in many easy cases, $L_{2}$ can be shown to be inequivalent to $L_{1}$.

Thus knots are determined by their complements, but links of more than one component in general are not. Three questions arise:

1) Can we characterize those links that are determined by their complements? That is, can we characterize those links $L_{1}$ such that, if $S^{3}-L_{1}$ is homeomorphic to $S^{3}-L_{2}$, then $L_{1}$ is equivalent to $L_{2}$ ?

2) For a given link (or a family of links) $L_{1}$, can we characterize the links $L_{2}$ such that $S^{3}-L_{1}$ is homeomorphic to $S^{3}-L_{2}$ ?

3) There are many interesting families of links which arise naturally in various contexts. (Boundary links, homology boundary links, pure links, Brunnian links; links of bounded genus, bridge number, braid index, unknotting number, etc) Is there any such family $\mathcal{L}$ such that if $L, L^{\prime} \in \mathcal{L}$ and $S^{3}-L$ is homeomorphic to $S^{3}-L^{\prime}$, then $L$ is equivalent to $L^{\prime}$ ?

For knots, of course, the Gordon-Luecke result answers all these questions. For links of more than one component, however, none of these questions have been addressed in the literature as far as we know.

We will define a class of links called HTB links, which is almost the same as the class of Brunnian links. We will show that for HTB links, the answer to Question 3 is yes (Theorem 3, our main result). Regarding Question 2, for an HTB link $L$, we will give a characterization of those $L^{\prime}$ with complement homeomorphic to that of $L$ (Theorem 4). With regard to Question 1, it follows from our answer to Question 3 and the twisting construction (mentioned above and discussed further below) that an HTB link is never determined by its complement (among all links) unless it is the trivial link or a knot.

Let $K_{1}$ and $K_{2}$ be two components of a link $L$. There is a well-defined linking number which indicates the homology class of $K_{1}$ in $S^{3}-K_{2}$. A link $L$ is homologically trivial if this linking number is 0 for any pair of components of $L$ (a property that does not depend on the orientations of $K_{1}$ and $K_{2}$ ). A link is trivial if the components bound pairwise disjoint disks. An $n$-component link is Brunnian if every $(n-1)$-component sublink is trivial. We consider an empty link to be trivial, and therefore we consider a knot to be a 1-component Brunnian link. A component of a link is unknotted if it bounds a disk in $S^{3}$, which may intersect the other components of the link. We define an HTB link to be a link that is both homologically trivial and Brunnian. The class of HTB links includes all Brunnian links with more than two components. 
In 1892, Brunn constructed links which become trivial when any single component is deleted, but there did not exist at the time rigorous methods to show that these links were in fact nontrivial. It was not until 1961 that it was shown by Debrunner [Deb61] (who also generalized Brunn's results) that nontrivial Brunnian links exist. Since that time, Brunnian links have become a standard subclass of links, and have been utilized and generalized in a number of ways.

Let $L$ be a link, and let $X_{L}$ be $S^{3}$ with an open tubular neighborhood $\eta(L)$ of $L$ removed. The boundary components of $X_{L}$ are two-dimensional tori, each torus associated to some component of $L$. Each torus separates $S^{3}$ into an inside (containing the associated component of $L$ ) and an outside. Each torus has a unique isotopy class of essential simple closed curve called the meridian which is homologically trivial inside of the torus. Likewise, each torus has a unique isotopy class of essential simple closed curve called the longitude which is homologically trivial outside the torus. The meridian and the longitude form a basis for $H_{1}$ of the torus.

The isotopy class of any unoriented simple closed curve on the torus is determined by a slope $r=p / q \in \mathbb{Q} \cup\{1 / 0\}$, where $p$ indicates how many times the curve wraps around in the meridian direction and $q$ indicates how many times it wraps around in the longitude direction. (Note that the specific orientations of the longitude and meridian are not important, but for $r$ to be well-defined they must together be oriented compatibly with the orientation of $S^{3}$.) Using the slope $r$, we may perform Dehn surgery on a component $K$ of a link by cutting out a solid torus neighborhood of $K$ from $S^{3}$ and regluing it so that the curve of slope $r$ now bounds a disk in the solid torus. Notice that if the slope is $1 / 0$ then performing Dehn surgery is simply removing a solid torus and replacing it the same way.

If $L$ is a link with $n$ components, then we will often write $L$ with an $n$-tuple after it. A slope $p / q$ in the $i$ th position will indicate that $p / q$ surgery has been performed on the $i$ th component, and a $*$ will indicate that no surgery has been performed. Sometimes, this notation will refer to the link formed by the components with a $*$ in the manifold resulting from the surgeries, and sometimes it will refer to the complement of this link. The meaning will be clear from the context. So, for example, $L=L(*, *, \cdots *)$, and $L$ with the first component deleted is $L(1 / 0, *, *, \cdots *)$, whereas $L(1 / 0, \ldots 1 / 0) \cong S^{3}$.

If $K$ is an unknotted component, then $1 / q$ Dehn surgery on $K$ gives the same result as cutting $S^{3}$ and the link along the disk bounded by $K$, twisting $q$ times, regluing, and deleting $K$. In particular, the link resulting from $1 / q$ surgery on an unknotted component of $L$ is a link in $S^{3}$ with one fewer component. 
As in [GL89], we will approach the proof of our main result by considering those Dehn surgeries on an HTB link $L$ which produce $S^{3}$. We start with the following theorem which, while no surprise to experts, does not seem to appear in the literature.

Theorem 1 If $L$ is a link with $n$ components and if $L\left(x_{1}, x_{2}, \ldots x_{n}\right) \cong S^{3}$ for every $n$-tuple $\left(x_{1}, x_{2}, \ldots x_{n}\right) \in\{1 / 0,-1,1\}^{n}$ then $L$ is a trivial link.

Proof We proceed by induction on $n$, the number of components of $L$. The theorem is true for knots in $S^{3}$ since, by the cyclic surgery theorem in [CGLS87], 1 and -1 surgery on a nontrivial knot cannot both produce a manifold with trivial fundamental group. Now, assuming the theorem is true for all links of $n-1$ or fewer components, we will show the result holds for any link with $n \geq 2$ components. Let $L=K_{1} \cup K_{2} \cup \cdots \cup K_{n}$ be a link in $S^{3}$ such that $L\left(x_{1}, x_{2}, \ldots x_{n}\right) \cong S^{3}$ for all $n$-tuples $\left(x_{1}, x_{2}, \ldots x_{n}\right) \in\{1 / 0,-1,1\}^{n}$

If $X_{L}=S^{3}-\eta(L)$ is reducible, then there is some $S^{2} \subset X_{L}$ which does not bound a ball. Since any $S^{2} \subset S^{3}$ separates and bounds a ball on each side, the sphere in $X_{L}$ must split $L$ into two nonempty sublinks $L_{1}$ and $L_{2}$. The inductive hypothesis implies that each of these sublinks is the trivial link. Furthermore, they are separated by a sphere, so their union $L$ is also the trivial link. In particular, $L$ is trivial if some torus $T_{i}$ on $\partial X_{L}$ has a compressing disk $D$ in $X_{L}$ because the frontier of a regular neighborhood of $T_{i} \cup D$ would be a reducing sphere. Therefore, we may assume that $X_{L}$ is irreducible with incompressible boundary.

Each component of $L$ must be unknotted because performing 1/0 surgery on any other component results in a link that, by induction, is trivial. Furthermore, each pair of distinct components $K_{i}$ and $K_{j}$ have $l k\left(K_{i}, K_{j}\right)=0$. Suppose that $l k\left(K_{1}, K_{2}\right)=m$. Let $K=L(1, *, 1 / 0,1 / 0, \ldots 1 / 0)$, a knot in $S^{3}$. Then $K\left(1-m^{2}\right)=L(1,1,1 / 0,1 / 0, \ldots 1 / 0)$ which is homeomorphic to $S^{3}$ by our assumption. See, for example, [Rol76] as a reference for this modification of surgery instructions. However, $H_{1}\left(K\left(1-m^{2}\right)\right) \cong \mathbb{Z} /\left(1-m^{2}\right) \mathbb{Z}$, so $m=0$. Therefore, the link resulting from 1 or -1 surgery on any one component is a link in $S^{3}$, and the meridian-longitude pairs of the remaining components are the same as the meridian-longitude pairs for the corresponding sublink of $L$.

Consider the links $L(-1, *, \ldots, *)$ and $L(1, *, \ldots, *)$. Each of these are $(n-1)-$ component links which satisfy the hypothesis of the theorem, so they are trivial by induction. Therefore, the torus $T_{2}=\partial \eta\left(K_{2}\right) \subset X_{L}$ becomes compressible after both 1 and -1 Dehn fillings on $T_{1} \subset \partial X_{L}$. Since $\Delta(1,-1)=2>1$ 
and since $X_{L}$ is irreducible with incompressible boundary, by Theorem 2.4.4 of [CGLS87] (generalized by $\mathrm{Wu}$ in [Wu92]) we see that $X_{L}$ is either $T^{2} \times I$ or a cable space, a solid torus with a regular neighborhood of a $(p, q), q \geq 2$, cable knot of the core curve of the torus removed.

In either case, $X_{L}$ is Seifert fibered. Burde and Murasugi produced a complete list in [BM70] of all links in $S^{3}$ with Seifert fibered complement. Any such link with more than one component has nontrivial linking number between some pair of components. This contradicts that every pair of components of $L$ are algebraically unlinked, so $X_{L}$ must in fact be reducible, proving the theorem.

Theorem 2 Let $L$ be an HTB link. Suppose there exist slopes $r_{i}=p_{i} / q_{i}$, such that $q_{i} \neq 0$ for all $i$, and such that $L\left(r_{1}, r_{2}, \ldots r_{n}\right)=S^{3}$. Then $L$ is trivial.

Proof When $L$ has one component, this is the well-known result of Gordon and Luecke. Suppose $L$ has $n>1$ components. Because $L$ is homologically trivial, its $i$ th meridian has order $\left|p_{i}\right|$ in $H_{1}\left(L\left(r_{1}, r_{2}, \ldots r_{n}\right)\right)=H_{1}\left(S^{3}\right)$. Thus $\left|p_{i}\right|=1$ for all $i$. The $n$th component of $L$ is unknotted because $L$ is Brunnian, and so $L\left(*, * \cdots *, 1 / q_{n}\right)$ is a link in $S^{3}$. The linking number of the $i$ th and $j$ th components of $L\left(*, * \cdots *, 1 / q_{n}\right)$ is $l_{i j}+q_{n} l_{i n} l_{j n}$, where $l_{i j}, l_{i n}$, and $l_{j n}$ are the linking numbers in $L$. (This holds for any link in which $q_{n}$ twists are made about an unknotted $n$th component.) Thus $L\left(*, * \cdots *, 1 / q_{n}\right)$ is homologically trivial.

Deleting the first component of $L\left(*, \cdots *, 1 / q_{n}\right)$ yields $L\left(1 / 0, *, \ldots, 1 / q_{n}\right)$, and since this link may also be obtained by deleting the first component of $L$ and then performing Dehn surgery on the last component of the resulting link, it is trivial. The same holds for the $i$ th component, $i<n$, and so $L\left(*, \cdots *, 1 / q_{n}\right)$ is Brunnian. Thus, by induction, $L\left(*, \cdots *, 1 / q_{n}\right)$ is trivial, and therefore $L\left(1 / x_{1}, \ldots 1 / x_{n-1}, 1 / q_{n}\right)=S^{3}$ for all integers $x_{1}, \ldots x_{n-1}$. Gordon and Luecke's result ([GL89]) then implies that $L\left(1 / x_{1}, \ldots 1 / x_{n-1}, *\right)$ is a trivial knot for all integers $x_{1}, \ldots x_{n-1}$, and therefore $L\left(1 / x_{1}, \ldots 1 / x_{n}\right)=S^{3}$ for all integers $x_{1}, \ldots x_{n}$. By Theorem $1, L$ is trivial.

Note that in the proof of Theorem 2 we use a weaker version of Theorem 1 , namely that the $x_{i}$ in the hypothesis range over all possible $1 / n_{i}$ slopes for any integers $n_{i}$, rather than just 0,1 , and -1 . In discussing a preliminary version of this work with Martin Scharlemann, we also discovered a proof of Theorem 2 that does not rely on Theorem 1 at all. Rather, the proof suggested 
by Scharlemann uses a theorem of Gabai concerning Dehn fillings of manifolds with taut foliations [Gab87] to complete the induction.

If the $i$ th component of a link $L$ is unknotted, then $L\left(*, *, \ldots 1 / q_{i}, *, \cdots *\right)$ is the link obtained by twisting $q_{i}$ times around that component and then discarding it. If $L$ is a nontrivial HTB link, then $L\left(*, *, \ldots 1 / q_{i}, *, \cdots *\right)$ cannot be a trivial link (unless $q_{i}=0$ ) because then $L\left(1,1, \ldots 1 / q_{i}, 1, \ldots 1\right.$ ) would be $S^{3}$, contradicting Theorem 2. This generalizes one result of Mathieu [Mat92], who showed that for a two-component HTB link, twisting around one component always ties a nontrivial knot in the other component. (More generally, he determines exactly when twisting around an unknotted disk in the complement of an unknot can produce a nontrivial knot.) Mathieu's result together with that of Gordon and Luecke implies Theorem 2 for two-component links.

We can now prove our main result.

Theorem 3 Let $L_{1}$ and $L_{2}$ be HTB links. Then $L_{1}$ is equivalent to $L_{2}$ if and only if $S^{3}-L_{1}$ is homeomorphic to $S^{3}-L_{2}$.

Proof Let $h: S^{3}-L_{1} \rightarrow S^{3}-L_{2}$ be a homeomorphism. (By a homeomorphism of link complements, we always mean an orientation preserving homeomorphism, as is required for link equivalence.) Since $L_{1}$ and $L_{2}$ are homologically trivial, the longitudes of the components of each link are null-homologous in the link complements. These are the only isotopy classes of essential simple closed curves on each boundary component of $S^{3}-\eta\left(L_{1}\right)$ and $S^{3}-\eta\left(L_{2}\right)$ that are null-homologous. Since $h$ induces isomorphisms on the homology groups, $h$ maps longitudes to longitudes. The map induced by $h$ on the homology of the torus boundaries of the complement of tubular neighborhoods of the $L_{i}$ must be invertible, and therefore the $i$ th meridian of $L_{1}$ must map to a slope $1 / q_{i}$ of the $i$ th component of $L_{2}$.

If every meridian of $L_{1}$ is taken to a meridian of $L_{2}$, then it is clear from the definition of Dehn surgery that we can extend $h$ to a homeomorphism $\hat{h}:\left(S^{3}, L_{1}\right) \rightarrow\left(S^{3}, L_{2}\right)$. That is, the two links are equivalent.

Assume without loss of generality that the first meridian of $L_{1}$ is taken to some slope $1 / q \neq 1 / 0$ of the first component of $L_{2}$. Then we can extend $h$ to a homeomorphism from $L_{1}(1 / 0, *, * \ldots *)$ to $L_{2}(1 / q, *, * \ldots *)$. The former is trivial because the link $L_{1}$ is Brunnian. Thus, the latter is also trivial since it is a link in $S^{3}$ and the trivial link is determined by its complement. Therefore, $L_{2}(1 / q, 1,1, \ldots 1) \cong S^{3}$ so $L_{2}$ must be the trivial link by Theorem 2 . As above, the homeomorphism $h$ implies that $L_{1}$ is also the trivial link, proving that $L_{1}$ and $L_{2}$ are equivalent. 
As mentioned at the beginning of the paper, one way to construct inequivalent links $L_{1}$ and $L_{2}$ with homeomorphic complements is to twist one or more times around an unknotted component $K$ of $L_{1}$ (without discarding $K$ ) to produce $L_{2}$. (See Figure 1.) More generally, a succession of such twisting operations may be performed, as long as there remains an unknotted component to twist around. It follows from Theorem 3 that if $L_{1}$ is an HTB link, then $L_{2}$ is never Brunnian and is therefore never equivalent to $L_{1}$. Moreover, if $L_{1}$ is an HTB link, then every $L_{2}$ with a homeomorphic complement is obtained this way. In contrast, Berge ([Ber91]) gives an explicit example of a pair of inequivalent 2 component links (not HTB links) with homeomorphic complements which remain inequivalent even after twisting along the unknotted components.
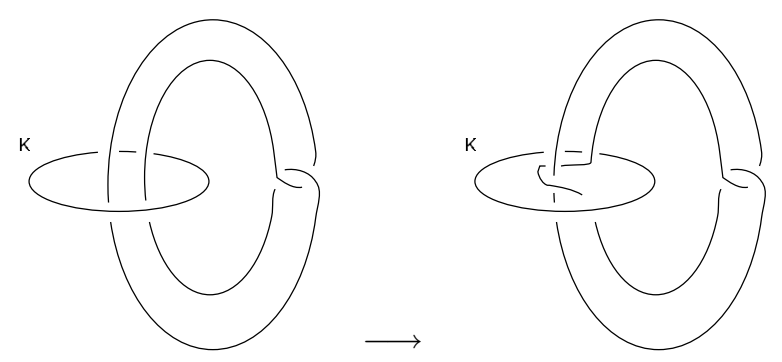

Figure 1: Inequivalent links formed by twisting along unknotted $K$

Theorem 4 Let $L_{1}$ be an HTB link and $L_{2}$ a link whose complement is homeomorphic to that of $L_{1}$. Then $L_{2}$ is obtained from $L_{1}$ by successive twists around unknotted components.

Proof Let $h: S^{3}-L_{1} \rightarrow S^{3}-L_{2}$ be a homeomorphism. Since $L_{1}$ is homologically trivial, the image of each longitude under $h$ must be an essential simple closed curve on the boundary of $S^{3}-\eta\left(L_{2}\right)$ that is null-homologous in the link complement. Therefore $L_{2}$ is also homologically trivial, and $h$ maps longitudes to longitudes.

As in the proof of Theorem 3 , the $i$ th meridian of $L_{1}$ must map to a slope $1 / q_{i}$ of the $i$ th component of $L_{2}$. Moreover, the $i$ th meridian of $L_{2}$ must then map under $h^{-1}$ to the slope $-1 / q_{i}$ of $L_{1}$. This implies that $L_{2}$ is determined by $L_{1}$ and the slopes $q_{i}$, for if there is a homeomorphism $h^{\prime}: S^{3}-L_{1} \rightarrow S^{3}-L_{2}^{\prime}$, then the composition $h^{\prime} \circ h^{-1}$ takes the meridians of $L_{2}$ to the meridians of $L_{2}^{\prime}$, so $L_{2}$ and $L_{2}^{\prime}$ are equivalent.

Thus the links with complements homeomorphic to that of $L_{1}$ are parameterized by $n$-tuples of slopes $\left(1 / q_{1}, 1 / q_{2}, \ldots 1 / q_{n}\right)$. If $q_{i} \neq 0$ for all $i$, then 
$L_{1}\left(-1 / q_{1}, \ldots,-1 / q_{n}\right) \cong S^{3}$ and by Theorem $2, L_{1}$ is trivial. Therefore, we assume that $q_{1}=0$. Since $L_{1}$ is Brunnian, it has a diagram where components $2,3, \ldots n$ are $n-1$ disjoint planar circles. Using this diagram, it is clear that we may perform $q_{i}$ twists on the $i$ th component for all $1<i \leq n$, and so in this way, we may realize any link $L_{2}$ whose complement is homeomorphic to $S^{3}-L_{1}$.

We now show how our results give ways of reducing the problem of deciding if a given $n$-component link is trivial to other decision problems. First of all, recognizing the trivial link may be reduced to recognizing $S^{3}$ itself, provided that our method of representing links and three-manifolds allows Dehn surgery to be done algorithmically. Algorithms to recognize $S^{3}$ were produced by Rubinstein in [Rub95] and Thompson in [Tho94].

Corollary 5 A link $L$ in $S^{3}$ with $n$ components is trivial if and only if $L\left(e_{1}, e_{2}, \ldots e_{n}\right)=S^{3}$ for each $\left(e_{1}, e_{2}, \ldots e_{n}\right) \in\{1 / 0,1\}^{n}$.

Proof We proceed by induction on the number of components in $L$. For knots, the corollary is true by [GL89]. Suppose that $L$ has more than one component. It must be homologically trivial by the argument in the proof of Theorem 1. Performing $1 / 0$ surgery on any one component results in a trivial link by induction, so $L$ is Brunnian. Since $L(1, \ldots, 1) \cong S^{3}$, L is trivial by Theorem 2.

If we take a recursive approach, we can reduce the problem of recognizing a trivial link to the problem of recognizing a trivial knot in $S^{3}$. Suppose we have Algorithm A which tells whether or not a standard knot diagram represents a trivial knot. Then we may use the next corollary to give an algorithm for recognizing a trivial link in $S^{3}$.

Corollary 6 A link $L$ with $n$ components is trivial if and only if

(1) $L$ is homologically trivial

(2) Each $(n-1)$-component sublink $L(*, * \ldots 1 / 0, \cdots *)$ is trivial.

(3) One of the $(n-1)$-component links $L(*, *, \ldots 1, \cdots *)$ is a trivial link in $S^{3}$.

Proof Conditions 1 and 2 imply that $L$ is an HTB link. If condition 3 is true, then $L(1, \ldots, 1) \cong S^{3}$ so $\mathrm{L}$ is trivial by Theorem 2 . 
Algorithm 7 An algorithm for recognizing a trivial link in $S^{3}$.

Given a diagram $D$ for a link $L$, the first step is to calculate the pairwise linking numbers of $L$. This is simply a matter of counting up positive and negative crossings in $D$. If any linking number is nonzero, then $L$ is not trivial. If all the pairwise linking numbers are zero, then pick a component $K$ of $L$ and check whether it is trivial using Algorithm A. If $K$ is not trivial, then $L$ is not trivial. If $K$ is trivial, then there exists a finite sequence of Reidemeister moves which transform $D$ into a diagram $D^{\prime}$ in which $K$ is represented by a simple closed curve with no self-crossings. Given any link diagram, there are only finitely many diagrams that result from a single Reidemeister move on that diagram. Produce all diagrams that result from a single Reidemeister move on $D$. Then produce all diagrams that result from a single Reidemeister move on any of these, and so forth. After finitely many steps, this will produce the diagram $D^{\prime}$. A twist around the disk bounded by $K$ may then be accomplished using the diagram $D^{\prime}$. We now need to check the triviality of $n+1(n-1)$-component links - namely the sublinks obtained by deleting 1 component from $L$ and the link $L(1 / 1, *, *, \cdots *)$. The algorithm then proceeds recursively.

This algorithm is not likely to be of much practical use. Even if we had an efficient Algorithm A, we don't know a bound on the number of Reidemeister moves it might take to convert a diagram with an unknotted component $K$ into a diagram where $K$ is represented by a circle. Moreover, each twist along such a circle is likely to raise the crossing number of the diagram substantially.

Suppose we have an $n$-unlink algorithm (ie, an algorithm for deciding whether an $n$-component link is trivial). Then we have an $(n-1)$-unlink algorithm as well, by adding an extra disjoint and unknotted component. What we have seen is that by applying twisting and deleting operations, we also have an $(n+1)$-unlink algorithm, and thus our $n$-unlink algorithm becomes an unlink algorithm for any number of components.

\section{Acknowledgment:}

The authors would like to thank Darren Long for very useful conversations regarding this work, and the referee for strengthening Theorem 1 and shortening its proof. The second author was partially supported by the US Naval Academy Research Council. 


\section{References}

[Ber91] John Berge. The knots in $D^{2} \times S^{1}$ which have nontrivial Dehn surgeries that yield $D^{2} \times S^{1}$. Topology Appl., 38(1):1-19, 1991 .

[BM70] Gerhard Burde and Kunio Murasugi. Links and Seifert fiber spaces. Duke Math. J., 37:89-93, 1970.

[CGLS87] Marc Culler, C. McA. Gordon, J. Luecke, and Peter B. Shalen. Dehn surgery on knots. Ann. of Math. (2), 125(2):237-300, 1987.

[Deb61] Hans Debrunner. Links of Brunnian type. Duke Math. J., 28:17-23, 1961.

[Gab87] David Gabai. Foliations and the topology of 3-manifolds. II. J. Differential Geom., 26(3):461-478, 1987.

[GL89] C. McA. Gordon and J. Luecke. Knots are determined by their complements. J. Amer. Math. Soc., 2(2):371-415, 1989.

[Mat92] Yves Mathieu. Unknotting, knotting by twists on disks and property (p) for knots in $S^{3}$. In Knots 90 (Osaka, 1990), pages 93-102. de Gruyter, Berlin, 1992.

[Rol76] Dale Rolfsen. Knots and links. Publish or Perish Inc., Berkeley, Calif., 1976. Mathematics Lecture Series, No. 7.

[Rub95] Joachim H. Rubinstein. An algorithm to recognize the 3-sphere. In Proceedings of the International Congress of Mathematicians, Vol. 1, 2 (Zürich, 1994), pages 601-611, Basel, 1995. Birkhäuser.

[Tho94] Abigail Thompson. Thin position and the recognition problem for $S^{3}$. Math. Res. Lett., 1(5):613-630, 1994.

[Wu92] Ying Qing Wu. Incompressibility of surfaces in surgered 3-manifolds. Topology, 31(2):271-279, 1992.

Barnard College, Columbia University

Department of Mathematics

New York, NY 10027, USA

and

New Mexico State University

Department of Mathematical Sciences

Las Cruces, NM 88003, USA

Email: mangum@math.columbia.edu, stanford@nmsu.edu

Received: 16 November 2000 